\title{
Implantação de uma Metodologia de Testes em Iterações para um Grupo Independente
}

\author{
Renata C. C. Diniz ${ }^{1}$, Juliana Ochner ${ }^{2}$ \\ ${ }^{1}$ SoftexRecife, - Recife - PE - Brasil \\ ${ }^{2}$ Centro de Informática \\ Universidade Federal de Pernambuco (UFPE) - Recife - PE - Brasil \\ rendiniz@gmail.com, jo@cin.ufpe.br
}

\begin{abstract}
This paper describes the implementation of a software test process for independent tests groups using iteration concepts. The goal of this experiment was apply the benefits of the iterations approach in order to simplify the definition and execution of a complete test process. This process was defined using as basis de Test discipline from RUP, the test artefact standard IEEE 829, practices defined on TMM and the Verification and Validation process areas from CMMI. The iterations had been executed following the priority and current situation of the team's process.
\end{abstract}

Resumo. Este artigo relata uma experiência de implantação de uma metodologia de testes em iterações para um grupo independente de testes. $O$ objetivo do experimento foi utilizar os benefícios da abordagem iterativa para facilitar a definição e implantação de um processo completo de testes. Este processo foi definido com base na disciplina Testes da metodologia de desenvolvimento de software RUP, do padrão para artefatos de testes IEEE 829, das práticas definidas pelo TMM e pelas áreas de processo Verificação e Validação do CMMI. As iterações foram executadas conforme a prioridade e situação atual dos processos da equipe.

\section{Introdução}

A indústria mundial de Tecnologia da Informação tem investido, cada vez mais, em atividades de testes. Carreiras de especialistas em testes vêm sendo criadas em maiores escalas. Empresas como AT\&T, Novell, Motorola e Datasul têm utilizado times independentes de testes no intuito de melhorar seu processo de testes e a qualidade do software produzido. Pesquisas realizadas pelo Software Testing Institute no ano de 2002 revelam que há uma tendência para que as empresas trabalhem com times de teste centralizados, independentes das equipes de desenvolvimento. [STI, 2002]

No contexto atual de alta competitividade, a descoberta e conseqüente remoção de defeitos através da implementação de processos de testes de software e da adoção e uso de ferramentas de suporte adequadas são competências mandatórias para empresas que buscam elevar a maturidade do seu processo de software e da qualidade dos seus produtos e serviços. 
Há uma tendência mundial em utilizar o conceito de maturidade para a implantação de processos. Nesta linha há o CMMI [CMMI, 2002], ISO 15504 [ISO/IEC, 1998], MPS.br [MPS.BR, 2006] e o TMM [TMM, 2002]: modelos de processo em maturidade, sendo o TMM focado no processo de testes. Por definição, modelos de processo definem requisitos para um processo efetivo [CMMI, 2002], mas o processo deve ser definido de acordo com as necessidades e disponibilidades de cada ambiente. Assim, uma empresa que deseje utilizar o CMMI ou o TMM deverá definir seu processo aderente a estes modelos, ou seja: atividades, artefatos, ferramentas, papéis e responsabilidades.

Diversas metodologias, ou processo, de desenvolvimento de software vêm sendo propostas, como: RUP [RUP, 1998] e Extreme Programming [XP, 1999], metodologia ágil surgida em 1999. Além de metodologias, existem normas e padrões propostos por diversas entidades no que tange o desenvolvimento de software. Para a área de testes especificamente é importante destacar o padrão 829 do IEEE [IEEE 829, 1998].

Apesar dos modelos e metodologias disponíveis, várias são as dificuldades encontradas na implantação de processos: resistência a mudanças, falta de recursos financeiros, conflito de prioridades entre projetos e implantação de processo, dificuldade em medir sucesso, benefícios geralmente obtidos a médio e longo prazo, muitas mudanças culturais e organizacionais em pouco tempo.

Cada vez mais, na indústria de software, o modelo iterativo vem se destacando como o mais apropriado e de melhor resultado para o ciclo de vida de desenvolvimento. Dentre os benefícios do modelo iterativo, estão: obtenção de pequenos resultados em curto prazo, melhor gerenciamento de riscos, feedback mais rápido, melhor entendimento das necessidades do cliente, possibilidade de priorização, melhor visibilidade do projeto [RUP, 1998].

Com o objetivo de facilitar a melhoria de processo de uma empresa realizamos a experiência de definir e implantar um processo de testes para um grupo independente em iterações. Este processo foi elaborado baseado no RUP e no padrão IEEE 829, buscando atender aos requisitos do CMMI para as áreas de processo Verificação e Validação até o nível 3 na representação contínua e ao TMM em seu nível 3. A partir do nível 4, estes modelos requerem controles estatísticos não abordados na implantação em questão.

\section{Contexto da Implantação de Metodologia de Testes}

O relato de experiência em questão irá descrever a implantação de uma metodologia de testes em iterações para um grupo independente de testes de uma empresa de software de Recife. Esta empresa possui um produto de software instalado em mais de 30 clientes no Brasil, com mais de 500 funcionalidades. Com a constante evolução deste produto devido à correção de bugs e melhorias incorporadas ao longo dos anos de uso, as atividades de testes seguiram aumentando, ou seja, cada vez necessitando de mais pessoas e mais tempo para serem executadas completamente.

A necessidade de controlar o esforço com testes e melhorar a qualidade do produto levou a empresa a criar um grupo independente de testes, separado da equipe de desenvolvimento. Este grupo gerou algumas melhorias para o produto, no entanto, o avanço ficou limitado devido à falta de processos e ferramentas. 
Esta empresa contratou o NEXT [NEXT], laboratório de testes vinculado ao SOFTEXRECIFE. O SOFTEX, Sociedade Brasileira para Promoção da Exportação de Software, é uma organização não governamental cujo objetivo social é de executar, promover, fomentar e apoiar atividades de inovação e desenvolvimento científico e tecnológico. O NEXT foi criado com o objetivo de aumentar a competitividade das empresas de TI, desenvolvendo capacitação e prestação de serviços, de forma inovadora, em testes de software e sistemas.

\section{Processo de Implantação de Metodologia de Testes}

O processo para definição e implantação de uma metodologia de testes para um grupo independente incluiu inicialmente um diagnóstico da situação atual e em seguida a elaboração e execução de um plano de ação em iterações.

\subsection{Diagnóstico da situação atual}

O projeto iniciou com um diagnóstico da situação atual do processo de testes. Um checklist foi elaborado com as melhores práticas de testes, baseado especialmente no RUP, no padrão IEEE 829, nas áreas de processo Verificação e Validação do modelo de maturidade CMMI e em práticas do TMM.

Este diagnóstico também abordou questões importantes de outros processos do desenvolvimento de software por afetarem fortemente o processo de testes. Dentre eles estão: Planejamento e Acompanhamento de Projetos, Requisitos e Gerência de Configuração.

O diagnóstico foi realizado por membros experientes em qualidade de software do SOFTEXRECIFE através de entrevistas aos membros da empresa, análise dos artefatos gerados e das ferramentas e dos sistemas utilizados. O resultado obtido foi apresentado para representantes da equipe de testes e, especialmente, a alta direção da empresa.

\subsection{Elaboração de um Plano de Ação}

Depois de identificado, através do diagnóstico, os pontos fortes e as oportunidades de melhoria do processo de testes, um Plano de Ação iterativo foi elaborado baseado nas boas práticas do TMM níveis 2 e 3 . A divisão das iterações seguiu as seguintes diretrizes:

- Priorizar a capacitação da equipe de testes;

- Priorizar atividades de testes independentes de outras equipes da empresa;

- Priorização da automação de testes;

- Iniciar práticas de planejamento de testes após a definição do processo operacional de testes;

- Definir atividades básicas, que dependem somente da área de testes, antes das avançadas, que estão relacionadas com outras áreas do desenvolvimento de software.

Com base nestas diretrizes, foi realizado um planejamento com 4 iterações e alguns pilotos. Dois tipos de piloto foram definidos no projeto:

- Piloto Exercício: este tipo de piloto teve como objetivo treinar a equipe nos processos definidos no âmbito da realidade da empresa. Os produtos produzidos durante estes pilotos não deveriam ser reaproveitados para os projetos, afim de 
que as pessoas se sentissem confortáveis para aprender, errar e corrigir. As atividades deste piloto não entraram nos cronogramas dos projetos em andamento.

- Piloto Real: este tipo de piloto utilizou os processos definidos em um projeto real. O objetivo deste piloto foi testar os processos definidos nos projetos reais. As atividades deste piloto fizeram parte dos cronogramas dos projetos em andamento.

Cada iteração foi iniciada com um treinamento específico sobre o escopo a ser tratado. Este treinamento propiciou que a equipe alinhasse o conhecimento e o vocabulário, de forma a facilitar a definição do processo específico para a empresa.

Um planejamento detalhado com datas, marcos, produtos a serem gerados foi elaborado no inicio de cada iteração e aprovado pela empresa. Cada iteração durou entre 3 e 4 semanas. Para balancear as atividades do novo processo e as atividades diárias da empresa, ficou definido que toda a equipe dedicaria $50 \%$ do expediente para o projeto de consultoria em testes e os outros $50 \%$ para as atividades dos projetos em andamento.

A definição de iterações subseqüentes se dava no decorrer do piloto exercício da iteração passada. Assim, toda a equipe de testes estava sempre ocupada e envolvida com o projeto. Durante todas as iterações foi dado um foco especial nas atividades para automação de testes, pois até o momento do projeto não era realizada pela equipe da empresa e por isso esta equipe despendia um esforço significativo com testes de regressão.

A figura abaixo exibe o ciclo de vida definido para o projeto



Figura 1 - Ciclo de vida da implantação de um processo de testes

\section{a) Iteração 1 - Práticas Básicas de Projetar Testes}

Objetivo: Definir como a equipe de testes irá projetar os testes, especialmente, como documentar casos de testes e massa de dados.

A equipe não projetava testes. Os testes eram realizados sem a especificação de casos de testes, procedimentos e massa de dados. Por ser esta uma atividade muito importante e que afetaria toda a cultura da equipe de testes, ela foi priorizada para a primeira iteração. Além disto, esta atividade não dependeria de nenhum outro setor da empresa, ou seja, poderia ser tratada e evoluída dentro da equipe de testes, conforme suas habilidades.

\section{Visão Geral do Processo:}

- Foram definidos os papéis e responsabilidades da equipe de testes: 
- Gerente de Teste: responsável pelo planejamento de testes e acompanhamento da equipe.

- Analista de Teste: responsável por projetar testes e executar testes manuais.

- Engenheiro de Teste: responsável por automatizar casos de testes e executá-los.

- Seleção, capacitação e uso da ferramenta de gerenciamento de testes TestLink [TESTLINK]: esta ferramenta foi selecionada a partir de uma análise de ferramentas freeware pelo NEXT. A equipe passou a documentar e gerenciar os casos de testes a partir desta ferramenta.

- Seleção, compra e instalação da ferramenta TestComplete [TESTCOMPLETE] para automação de teste, devido a ótima relação custo $\mathrm{x}$ benefício deste produto. Por não ser uma atividade trivial, foi adotado que a automação de testes seria realizada em todas as iterações do projeto para que a equipe se especializasse nesta atividade.

- Massa de dados: foi definida uma metodologia e forma de especificação da massa de dados de testes. Esta massa de dados passou a ser documentada em um banco de dados.

- Priorização de casos de testes: definição de uma metodologia para priorizar casos de testes conforme freqüência de uso do respectivo cenário, probabilidade de gerar falhas e efeito das falhas. Esta priorização contribui no planejamento de suítes de testes. A priorização dos casos de testes resultante passou a ser registrada na ferramenta TestLink.

- Definição de guias para especificação de casos de testes com lições aprendidas e boas práticas.

\section{b) Iteração 2 - Práticas Básicas de Execução e Análise de Testes}

Objetivo: Definir como a equipe irá inspecionar o projeto de testes, além de executar e reportar os resultados de testes. Nesta iteração foi incluída uma primeira atividade com envolvimento de outros grupos da empresa além do grupo de testes: a participação dos analistas de requisitos na inspeção do projeto de testes.

\section{Visão Geral do Processo:}

- Definição do processo de inspeção para que os analistas de sistemas e a equipe de testes inspecionem o projeto de testes elaborado. Com esta inspeção, os analistas de sistemas, especialistas nos requisitos do sistema, passaram a contribuir para a elaboração do projeto de teste, garantindo assim uma boa cobertura dos casos de testes elaborados e conformidade destes com os requisitos.

- Definição de suítes e rodadas de testes, além de registro da execução dos testes na ferramenta de gerenciamento de testes TestLink.

- Definição de como documentar defeitos encontrados, ou seja, quais características dos defeitos reportar, como: descrição do defeito encontrado, severidade, anexos com telas de comprovação do defeito, categoria (bug ou sugestão de melhoria), passos para reprodução.

- Definição de um Relatório de Testes a ser elaborado pelo gerente de testes ao final dos testes pré-release. 
- Definição de medições iniciais, retiradas diretamente da ferramenta de gerenciamento de testes, como:

- Quantidade de casos de testes planejados x realizados

- Quantidade de problemas encontrados

- Cobertura dos testes em relação aos requisitos

\section{c) Iteração 3 - Práticas Avançadas de Planejamento de Testes}

Objetivo: A partir do momento que a equipe já definiu e aprendeu a desempenhar as atividades básicas de testes (projetar, inspecionar, executar e reportar resultados de testes), a iteração 3 passou a abordar a definição de um planejamento de testes.

\section{Visão Geral do Processo:}

- Revisão dos requisitos: para planejar as atividades de testes é importante entender os requisitos do projeto, complexidade, correções do produto no escopo.

- Definição de um Plano de Testes contendo: marcos, requisitos a serem testados, suítes de testes, tipos de testes, compromissos, critério de aceitação, papéis e responsabilidades.

- Definição de diretrizes para elaboração do cronograma de testes

- Melhorias no controle de configuração para controle das versões testadas e problemas encontrados.

- Definição de processo de acompanhamento com elaboração de um relatório periódico com status das atividades da equipe de testes.

- Definição de método de estimativa de esforço de testes com base na quantidade e complexidade dos requisitos

- Métricas de produtividade e qualidade do produto (incluindo análise de satisfação do cliente)

- Definição de como identificar, documentar e disseminar lições aprendidas

\section{d) Iteração 4 - Práticas Avançadas de Requisitos}

Objetivo: Definir e implementar práticas de requisitos não incorporadas pela empresa, como especificação de requisitos não funcionais para viabilizar a definição de testes para estes tipos de requisitos.

\section{Visão Geral do Processo:}

Estava previsto as seguintes ações para esta iteração:

- Definição de Requisitos Não Funcionais;

- Projeto de testes de Requisitos Não Funcionais

- Seleção de uma ferramenta de testes para execução de testes de requisitos Não Funcionais;

- Automação de testes de Requisitos Não Funcionais;

- Rastreabilidade de requisitos e análise de impacto.

A empresa solicitou excluir do escopo do projeto estas ações, pois estava implantando, junto com uma consultoria especializada, o MPS.BR nível G, o qual requer a definição dos requisitos não funcionais e práticas de rastreabilidade. Desta forma, a empresa optou por evoluir os testes desta área após a certificação, quando o processo de requisitos já estiver maduro. Esta iteração não foi realizada. 


\section{Resultados Obtidos}

Dentre os aspectos positivos da implantação de uma metodologia de testes de forma iterativa, se faz importante destacar:

- Envolvimento da equipe completa de testes desde as semanas iniciais do projeto. Em um projeto tradicional de definição de processo, geralmente há uma fase de definição de processo junto com alguns representantes da empresa e outra fase piloto onde o restante da equipe é envolvida. Neste projeto, após a definição do processo da iteração 1 , todos da equipe foram envolvidos no projeto através da participação nos pilotos exercícios e no piloto real;

- Entendimento da cultura e habilidades da equipe desde o inicio do projeto. Já com a realização do primeiro piloto-exercício, após 4 semanas de projeto, foi possível conhecer a fundo a cultura, necessidades, dificuldades e competências da equipe e da empresa, o que permitiu que a definição do processo fosse baseada neste conhecimento e, portanto, mais próximo da realidade $\mathrm{e}$ necessidades da empresa. Além disto, foi possível antecipar problemas e tratálos de forma a não atrasar o projeto.

- Apresentação de resultados para a equipe de testes e para toda a empresa em um curto espaço de tempo, o que aumentou a motivação da equipe e a confiança no projeto. Como as iterações duraram entre 3 e 4 semanas, ao fim de cada uma, era apresentado para toda a empresa o que foi realizado e produzido, as lições e constatações obtidas.

- Definição do processo de planejamento de testes a partir de um conhecimento prévio das atividades de testes e desempenho da equipe. O planejamento dos testes foi definido com um entendimento mais profundo acerca das atividades de testes. Por isto, ficou bem mais fácil definir métricas, método de estimativa, e conteúdo do planejamento.

- Incorporação de lições aprendidas.

- Separação entre os papéis dos membros da equipe, Engenheiros e Analistas de Testes, o que possibilitou a evolução rápida da automação dos testes reduzindo o esforço gasto para execução dos testes de regressão.

- Possibilidade de treinamento e capacitação da equipe nos processos aos poucos aproximando a prática da teoria. Sabe-se da dificuldade comum em assimilar todos os conceitos de um treinamento pronto aplicá-los na realidade de cada um. Para transpor esta barreira, foram dados treinamentos específicos no escopo da iteração e poucas semanas depois a equipe tinha que colocar em prática o que foi ensinado nos pilotos-exercício. Esta estratégia contribuiu fortemente para assimilação das práticas definidas no projeto.

Para se beneficiar do modelo iterativo para implantação de uma metodologia, algumas ações se mostraram essenciais, como:

- Necessidade de planejamento e acompanhamento detalhado e formalizado;

- Importância dos treinamentos iniciais;

- Aumento no tempo total do projeto, já que vários pilotos-exercícios foram executados. 


\section{Conclusão}

Este artigo apresentou a implantação de uma metodologia de testes para um grupo independente de testes em iterações. Cada iteração foi realizada em um período de 34 semanas e contou com um escopo bem definido, treinamentos e pilotos específicos. As iterações foram planejadas baseadas no modelo TMM e de forma a propiciar mais rapidamente resultados para a empresa.

$\mathrm{O}$ experimento apresentou bons resultados e mostrou como a empresa foi beneficiada por esta abordagem, podendo assim concluir que a metodologia utilizada é viável de ser aplicada para um grupo independente de testes. A metodologia definida possibilitou estruturar a área de testes, definir o relacionamento com outras áreas e melhorar a qualidade do produto gerado.

\section{Referências}

[CMMI, 2002] CMMI for Software Engineering, Version 1.1, Continuous Representation (CMMI-SW, V1.1, Continuous), Technical Report CMU/SEI-2002TR028, Agosto de 2002.

[IEEE 829, 1998] The Institute of Electrical and Electronics Engineers. IEEE Std 829: Standard for Software Test Documentation. New York: IEEE Computer Society, September, 1998.

[RUP, 1998] P. Krunchten, The Rational Unified Process: An introduction, Addison Wesley, 1998

[ISO/IEC 1998] The International Organization for Standardization and The International Electrotechnical Commission, ISO/IEC TR 15504 Software Process Assessment.1998.

[MPS.BR, 2006] Associação para Promoção da Excelência do Software Brasileiro SOFTEX. MPS.BR - Modelo de Referência, versão 1.1, maio 2006. Disponível em: http://www.softex.br/

[NEXT] Núcleo em Excelência em Testes de Software, http://www.next.org.br/. Acessado em 02/04/2007

[STI, 2002] Software Testing Institute website, http://www.softwaretestinginstitute.com/, Acessado em 02/04/2007.

[TESTCOMPLETE] Automated QA Test Complete.

http://www.automatedqa.com/products/testcomplete/. Acessado em 02/04/2007

[TESTLINK] TestLink. http://www.teslink.org. Acessado em 31/01/2007

[TMM, 2002] E, Veenendaal and R, Swinkels, Guidelines for Testing Maturity, publicado em Professional Tester, Volume Three, Issue No.1, March 2002.

[XP, 1999] K. Beck, Exteme Programming Explained: Embrace change, AddisonWesley, 1999. 